\title{
Nautisch-militärische Technologie beim vierten Kreuzzug 1202-1204
}

\author{
Rouven Thäwel
}

Der Vierte Kreuzzug war eine Konsequenz aus dem fehlgeschlagenen Dritten Kreuzzug, der sein Ziel, die Rückeroberung der an die Muslime verlorengegangenen Stadt Jerusalem, nicht hatte erreichen können. So rief Innozenz III. kurz nach seiner Wahl zum Papst im Jahr 1198 zum erneuten Kreuzzug auf. Doch auch dieser Kreuzzug sollte dem Ziel Jerusalem nicht einmal nahekommen und endete stattdessen mit der Eroberung und Plünderung der Hauptstadt des christlichen Kaiserreiches Byzanz, Konstantinopel.

Im Fokus der historischen Forschung lag lange Zeit vor allem die Frage nach den Ursachen dieses folgenschweren Kurswechsels der Unternehmung. Weniger Beachtung fand der Einsatz der militärischen und nautischen Technologie während der zweimaligen Eroberung Konstantinopels in den Jahren 1203 und 1204. Dieser Beitrag wirft einen Blick auf die Zusammensetzung der beteiligten Streitkräfte sowie auf die von ihnen während der Kampfhandlungen verwendete Militärtechnologie und ihre Bedeutung für den Ausgang des Vierten Kreuzzugs. Mit den Aufzeichnungen der Kreuzfahrer Geoffroy de Villehardouin und Robert de Clari stehen zwei Augenzeugenberichte zur Verfügung, die den Kampf um Konstantinopel eindrücklich darstellen. Villehardouin berichtet aus der Perspektive einer der Führungspersönlichkeiten des Kreuzzugs, Clari aus der eines einfachen Ritters. Auf byzantinischer Seite schildert der Adlige Niketas Choniates die Ereignisse aus seiner Sicht.

In Westeuropa wurden die Streitkräfte hauptsächlich von den Feudalherren gestellt. Sie waren diejenigen, welche über die nötigen Ressourcen verfügten, um sich selbst und weitere Truppen auszurüsten und zu bewaffnen. Den Kern der Heere bildete die schwere Kavallerie in Form der stark gerüsteten Ritterschaft. ${ }^{1}$ In dem letzten Jahrzehnt vor dem Vierten Kreuzzug hatte die Panzerung der europäischen Ritter, vor allem die Gesichtspanzerung, im Vergleich zur bisherigen Rüstung stark zugenommen, was wohl auf die Neuerungen in der Waffentechnologie zurückzuführen

\footnotetext{
1 Taxiarchis G. Kolias: Military Aspects of the Conquest of Constantinople by the Crusaders, in: Angeliki Laiou (Hg.): Urbs Capta. The Fourth Crusade and its Consequences/La IVe Croisade et ses Conséquences (Réalités Byzantines 10), Paris 2005, S. 123-138, hier S. 123, im Folgenden zitiert als Kolias: Aspects.
} 
ist, die sich in den letzten Dekaden des 12. Jahrhunderts entwickelt hatten. ${ }^{2}$ Obwohl es den niederen Klassen offiziell weiterhin verboten war Waffen zu tragen, gab es, gerade in Frankreich, immer mehr im Umgang mit Waffen geübte Söldner, die schon beinahe eine eigene Klasse bildeten. Die Armeen wurden dadurch immer professioneller. Besonders das flämische Kontingent des Kreuzfahrerheeres bestand zu einem Großteil aus Söldnern. In Flandern hatte eine in Verbindung mit einem rasanten ökonomischen Aufschwung entstandene Urbanisierung und das Turnierverbot durch die Kirche der Ritterschaft immer weniger Grund gegeben sich im Kriegshandwerk zu üben. Finanzielle Nöte leiteten zudem in Flandern bereits Ende des 12 . Jahrhunderts den allmählichen Niedergang der Ritterschaft ein. ${ }^{3}$

Besonders eine Waffe erfuhr zu dieser Zeit eine rasante Entwicklung und Verbreitung: die Armbrust, eine meistens aus Holz hergestellte Fernkampfwaffe. Die Sehne, die sich in horizontaler Lage an der Spitze befand, wurde durch Muskelkraft mit den Händen oder durch einen Kurbelmechanismus gespannt und in eine Nut aus Horn oder anderem harten Material eingespannt. Durch einen Abzug, ähnlich dem moderner Feuerwaffen, wurde das geladene Geschoss freigelassen. Die Armbrust verschoss, anders als der Bogen, keine Pfeile, sondern Bolzen. Diese waren etwa $30 \mathrm{~cm}$ lang und 1,25cm dick mit einer eisernen, meist pyramidal geformten Spitze. Obwohl die Schussfrequenz deutlich langsamer war als die des Bogens, lag der Vorteil der Armbrust darin, dass deutlich weniger Übung für die Handhabung dieser Waffe nötig war. Ihre Reichweite lag zwischen 350 und 500 Metern. Die Wirkung der Armbrust war so verheerend, dass die Kirche den Gebrauch offiziell verbot, was jedoch kaum Beachtung fand. ${ }^{4}$ Villehardouin verwendet in seinem Bericht des Öfteren Armbrustschussweiten als Längenangaben, ${ }^{5}$ was die Verbreitung und die Bedeutung der Waffe illustriert. Gerade in der Belagerungskriegsführung waren Armbrustschützen in den letzten Jahrzenten vor dem Vierten Kreuzzug immer wichtiger geworden ${ }^{6}$ und häufig wurden ausschließlich aus solchen Schützen bestehende

\footnotetext{
2 David Nicolle: The Fourth Crusade. The Betrayal of Byzantium (Campaign 237), Oxford 2011, S. 29, im Folgenden zitiert als Nicolle: Crusade.

3 Nicolle: Crusade, S. 30f.

${ }^{4}$ Robert D. Smith: Art. Weapons, Missile, in: The Oxford Encyclopedia of Medieval Warfare and Military Technology 3, Oxford 2010, S. 441-445, hier S. 444, im Folgenden zitiert als Smith: Art. Weapons.

${ }^{5}$ Geoffroy de Villehardouin: Die Eroberung von Konstantinopel, in: Chroniken des Vierten Kreuzzugs. Die Augenzeugenberichte von Geoffroy de Villehardouin und Robert de Clari, ins Neuhochdeutsche übersetzt, eingeleitet und erläutert von Gerhard E. Sollbach (Bibliothek der Historischen Forschung 9), Pfaffenweiler 1998, S. 20-81, hier S. 58, im Folgenden zitiert als: Geoffroy de Villehardouin: Eroberung.

${ }^{6}$ Kolias: Aspects, S. 130.
} 
Einheiten als Söldner angeheuert. ${ }^{7}$

Ein gänzlich anderes Bild lieferten die Streitkräfte des Stadtstaates Venedig. Seine auf eine Kombination aus See- und Landkriegsführung spezialisierte Armee bestand aus effektiven und hochmotivierten freien Städtern, die sich oft in einer Miliz zusammenschlossen. Dafür gab es in Venedig keine typische Ritterschaft. Dies führte dazu, dass auch fähige Personen aus den unteren sozialen Schichten militärische und technologische Führungspositionen besetzten. ${ }^{8}$ Auch die Ruderer auf den Galeeren waren freie Bürger, die penibel ausgewählt wurden. ${ }^{9}$ Seine Heimatstadt auf einem der Kriegsschiffe Venedigs verteidigen zu dürfen, wurde als hohe Ehre angesehen. Venedig hatte als Seefahrernation einen hohen Kenntnisstand was nautische Technologie betraf. Die Schiffe der venezianischen Flotte wurden für gewöhnlich in Partnerschaften gebaut und die erste Reise sollte die Baukosten decken, für die oft Geld geliehen werden musste. Sicherlich lag auch hier ein immenser Motivationsgrund der am Kreuzzug teilnehmenden Venezianer. ${ }^{10}$

Nach den Niederlagen gegen die Seldschuken 1071 bei Manzikert und 1176 bei Myriokephalon war ein Rückgang der militärischen Stärke Byzanz' zu verzeichnen. Allerdings sollte dieser nicht übertrieben hervorgehoben werden, da Byzanz immer noch über große militärische Ressourcen verfügte. Diese reichten aus, um den normannischen Eroberungsdrang während des 12. Jahrhunderts zu stoppen. Allerdings trifft es zu, dass Byzanz zur Zeit des Vierten Kreuzzugs relativ arm an Bevölkerung und Arbeitskraft war, was dazu führte, dass man verstärkt auf ausländische Söldner zur Verteidigung zurückgreifen musste. Auch Lateiner dienten in der Armee Konstantinopels, so zum Beispiel Genueser und Pisaner. ${ }^{11}$

Das prominenteste Beispiel der Söldnertruppen auf byzantinischer Seite ist wohl die Warägergarde. Sie war die Elitegarde des byzantinischen Kaisers und rekrutierte sich ursprünglich aus skandinavischen und angelsächsischen Einwanderern. ,Waräger' war im byzantinischen Griechisch die Bezeichnung für alle, die aus NordWesteuropa, über das Land der Rus, nach Byzanz kamen. ${ }^{12}$ Als persönliche

\footnotetext{
${ }^{7}$ Smith: Art. Weapons, S. 444.

${ }^{8}$ Nicolle: Crusade, S. 32.

${ }_{9}^{9}$ John D. Dotson: Art. Galleys, in: The Oxford Encyclopedia of Medieval Warfare and Military Technology 2, Oxford 2010, S. 146-148, hier S. 146.

${ }^{10}$ Nicolle: Crusade, S. 32f.

11 Nicolle: Crusade, S. 33f.

${ }^{12}$ Stephen Turnbull: The Walls of Constantinople AD 324-1453 (Fortress 25), 4. Aufl., Oxford 2008, S. 35f., im Folgenden zitiert als Turnbull: Walls.
} 
Leibwache des Kaisers hatte die Warägergarde ihr Quartier direkt am Blachernenpalast. ${ }^{13}$ Ihre Mitglieder waren vor allem berüchtigt durch den Einsatz ihrer bevorzugten Waffe, einer langstieligen Doppelaxt. ${ }^{14}$

Die restlichen byzantinischen Landstreitkräfte setzten sich größtenteils aus dem bewaffneten Fußvolk zusammen, zu dem auch Bogen- und Armbrustschützen und die Bedienungsmannschaften der Kriegsmaschinen gehörten. Der Anteil der schweren Reiterei war wahrscheinlich kleiner als im Heer der Kreuzfahrer, dafür verfügte die kaiserliche Armee über mehr leichte Kavallerie. ${ }^{15}$ Während der Belagerung von Konstantinopel scheint sich auch ein Teil der Zivilbevölkerung an der Verteidigung beteiligt zu haben. ${ }^{16}$ Um die Kontrolle über sein Herrschaftsgebiet zu gewährleisten, setzte Byzanz vor allem auf ein System aus Festungen und befestigten Städten. Mit der Bedeutung solcher Schlüsselpositionen war auch die der Belagerungsstrategien gestiegen. ${ }^{17}$

Woran es Konstantinopel vollkommen fehlte, war eine einsatzbereite Flotte. Diese war in den letzten Regierungsjahren Alexios III. zunehmend heruntergekommen. Choniates beschuldigt den Kaiser, zugelassen zu haben, dass der Flottenführer, Michael Stryphnos, der seine Position hauptsächlich der Tatsache zu verdanken gehabt hätte, dass er der Schwager der Kaiserin war, das „verschacherte“, was von der byzantinischen Marine noch übrig war. ${ }^{18}$ Besonders die Kriegsgaleeren benötigten gut ausgebildete und an die schwere Ruderarbeit gewöhnte Besatzungen, aber die generelle Vernachlässigung der Flotte hatte innerhalb weniger Jahre zur beinahe völligen Auflösung ihrer alten Schlagkraft geführt. ${ }^{19}$ Als die Kreuzfahrer Konstantino-

\footnotetext{
${ }^{13}$ Jonathan Shepard: Art. Varangian Guard, in: The Oxford Encyclopedia of Medieval Warfare and Military Technology 3, Oxford 2010, S. 396-398, hier S. 397.

${ }^{14}$ Kolias: Aspects, S. 130.

15 Kolias: Aspects, S. 128-130.

16 Robert de Clari: Die Eroberung von Konstantinopel, in: Chroniken des Vierten Kreuzzugs. Die Augenzeugenberichte von Geoffroy de Villehardouin und Robert de Clari, ins Neuhochdeutsche übersetzt, eingeleitet und erläutert von Gerhard E. Sollbach (Bibliothek der Historischen Forschung 9), Pfaffenweiler 1998, S. 82-137, hier S. 98, im Folgenden zitiert als Robert de Clari: Eroberung.

17 Kolias: Aspects, S. 124.

${ }^{18}$ Niketas Choniates: Die Kreuzfahrer erobern Konstantinopel, in: Die Regierungszeit der Kaiser Alexios Angelos, Isaak Angelos und Alexios Dukas, die Schicksale der Stadt nach der Einnahme sowie das „Buch von den Bildsäulen“ (1195-1206) aus dem Geschichtswerk des Niketas Choniates, je übersetzt, eingeleitet und erklärt von Franz Gabler, 2. unveränderte Aufl., Graz u.a. 1958, S. 17-230, hier S. 115, im Folgenden zitiert als Niketas Choniates: Kreuzfahrer.

${ }^{19}$ John H. Pryor: The Venetian Fleet for the Fourth Crusade and the Diversion of the Crusade to Constantinople, in: Marcus Bull; Norman Housley (Hgg.): The Experience of Crusading, Bd. 1, Cambridge 2003, S. 103-123, hier S. 109, im Folgenden zitiert als Pryor: Fleet.
} 
pel 1203 erreichten, standen lediglich noch zwanzig vollkommen überholungsbedürftige Schiffe zur Verfügung, um die Stadt und ihren Hafen zu verteidigen. ${ }^{20}$

Die Anstrengungen, die zur Vorbereitung des Vierten Kreuzzuges unternommen wurden, waren gewaltig. In Venedig wurden für drei Jahre sämtliche anderen Tätigkeiten untersagt und die Stadt konzentrierte sich einzig darauf, die für das Unternehmen notwendige Flotte zu bauen. Der zwischen den Kreuzfahrern und Venezianern geschlossene Vertrag sah vor, dass letztere genügend Schiffe zum Transport von 4.500 Rittern, ebenso vielen Pferden, 9.000 Knappen und 20.000 Fußsoldaten, samt deren anfallender Verpflegung für ein Jahr zu stellen hatten. Des Weiteren versprach Venedig zusätzliche 50 Kriegsgaleeren auf eigene Kosten zu bauen, die den Kreuzzug ebenfalls antreten sollten. ${ }^{21}$

Die venezianische Galeere war ein etwa 38m langes, schnelles Kriegsschiff, das sowohl mit einem dreieckigen Segel als auch durch Ruderkraft angetrieben wurde. ${ }^{22}$ Dieselbe Antriebstechnik hatten auch die huissiers, die Transportschiffe, in denen die Pferde und Knappen unterkamen. Sie hatten spezielle Heckaufbauten, aus denen eine Brückenkonstruktion herabgelassen werden konnte, um das Laden und Entladen der Pferde zu erleichtern. Diese Aufbauten ermöglichten außerdem die Verwendung der huissiers als Landungsbote für amphibische Militäraktionen. ${ }^{23}$ Die übrigen Schiffe der Flotte waren große Segelschiffe, von denen jedes bis zu 600 Passagiere aufnehmen konnte. Sie waren für den Transport der Ritter und des übrigen Fußvolks vorgesehen. ${ }^{24}$

Der personelle Aufwand Venedigs stand dem der Kreuzfahrer in nichts nach, denn neben den Schiffen selbst, stellte es auch deren Besatzungen. Die Mannschaft einer vollbesetzten Galeere war ca. 152 Mann stark, was allein für die 50 Kriegsschiffe eine Mannschaftsstärke von 7.600 bedeutet. Um die huissiers zu bemannen, waren nur etwa 130 Mann nötig. Da jedes aber nur 30 Pferde laden konnte, wurden für die 4.500 Reittiere der Kreuzfahrer 150 dieser Transportschiffe benötigt und damit

\footnotetext{
20 John H. Pryor; Peter R. Wilson: The Chain of the Golden Horn, 5-7 July 1203, in: Iris Shagrir; Ronnie Ellenblum; Jonathan Riley-Smith (Hgg.): In Laudem Hierosolymitani. Studies in Crusades and Medieval Culture in Honour of Benjamin Z. Kedar, Bd. 1, Hampshire u.a. 2007, S. 369-384, hier S. 384, im Folgenden zitiert als Pryor; Wilson: Chain.

${ }^{21}$ Geoffroy de Villehardouin: Eroberung, S. 24.

22 Susan Rose: Art. Ships and Sailing, in: The Oxford Encyclopedia of Medieval Warfare and Military Technology 3, Oxford 2010, S. 256-260, hier S. 259.

23 Pryor: Fleet, S. 103.

${ }^{24}$ Pryor: Fleet, S. 116.
} 
auch ca. 19.500 Mann Besatzung. Für die Segelschiffe, die die übrigen 24.500 Passagiere beförderten, müssen noch einmal etwa 4.500 erforderliche Matrosen geschätzt werden. Damit musste das venezianische Kontingent über 31.000 Mann stark gewesen sein und kam dem der Kreuzfahrer in etwa gleich. ${ }^{25}$

Die Flotte stach im Oktober 1202 in See und erreichte am 10. November Zara ${ }^{26}$, welches, obwohl eine christliche Stadt, sofort belagert wurde und am 24. November kapitulieren musste. Das Heer überwinterte in Zara. Während der Ruhepause stieß der Sohn des durch Alexios III. gestürzten ehemaligen byzantinischen Kaisers Isaak Angelos II. zu den Kreuzfahrern. Der später als Alexios IV. bekannte junge Mann bot den Kreuzfahrern eine hohe Belohnung, sollten sie ihm helfen den byzantinischen Thron zurückzugewinnen. Unter anderem versprach er laut Villehardouin, dass Byzanz sich wieder der katholischen Kirche und dem Papst unterwerfen würde, sowie 200.000 Silbermark, Lebensmittel für ein Jahr und 10.000 Mann, die den weiteren Kreuzzug unterstützen würden. ${ }^{27}$ Der Vorschlag wurde trotz teils heftigem Widerstand in den Reihen der Kreuzfahrer angenommen und so setzte die Flotte im April 1203 Segel in Richtung Konstantinopel, das sie schließlich am 24. Juni erreichte.

Die Hauptstadt des byzantinischen Reiches erstreckte sich Anfang des 13. Jahrhunderts über eine spitz zulaufende Landzunge auf der europäischen Seite des Bosporus, die in dessen südliche Mündung hineinragt. Die Stadt war an drei Seiten von Wasser umgeben. Im Süden grenzte sie an das Marmarameer, das am sich zuspitzenden östlichen Ende der Stadt in die Meerenge überging. Nördlich verlief das Goldene Horn, eine breite Flussmündung, die ebenfalls in den Bosporus hineinfloss und an dessen nördlichem Ufer die genuesische Handelskolonie Pera lag. Dem Verlauf der Halbinsel folgend, verbreiterte sich Konstantinopel in Richtung Westen. Alle Seiten wurden durch hohe Mauern geschützt.

Das Kreuzfahrerheer landete auf der asiatischen Seite des Bosporus und am 26. Juni marschierten sie an der Küste entlang gegen Konstantinopel, auf dem Meer begleitete die Flotte die Truppenbewegungen. Choniates berichtet von einem eher halbherzigen oder sogar unfähigen Widerstand der Byzantiner: „Aus der Ferne schossen die Rhomäer auf die Schiffe, teils von den Hügeln hinab, teils von der Küste aus, aber die Geschosse hatten keine Wirkung, die meisten reichten nicht einmal so weit und

\footnotetext{
25 Pryor: Fleet, S. 116-119.

26 Heute: Zadar, Kroatien.

27 Geoffroy de Villehardouin: Eroberung, S. 41.
} 


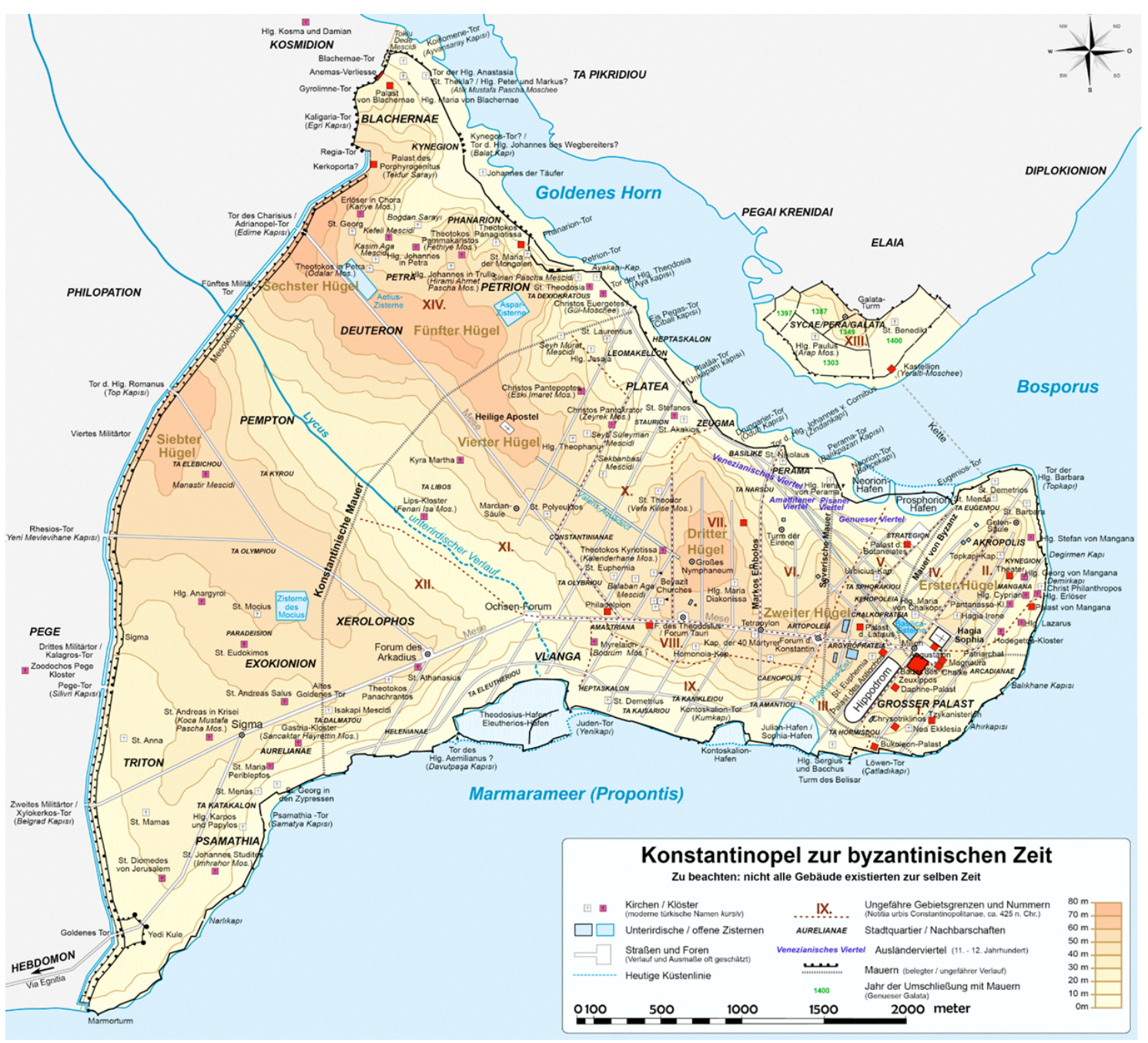

Abb. 1: Stadtgebiet von Konstantinopel im Mittelalter (wikimedia commons, Autor: Cplakidas, https://commons.wikimedia.org/wiki/File:Byzantine_Constantinople-en.svg (12.07.2021)).

fielen ins Meer. Ein anderer rhomäischer Heerhaufen lagerte oberhalb von Damatrys, um Landungsversuche der Reiter zu verhindern. Aber auch diese Leute waren zu nichts nütze. ${ }^{28}$ Das erste große Hindernis für die Kreuzfahrer war der befestigte Turm von Galata. Auf der Konstantinopel gegenüberliegenden Seite des Goldenen Horns gelegen, spannte sich von hier eine massive Eisenkette bis nach Konstantinopel, die jedem feindlichen Schiff die Einfahrt in den Hafen unmöglich machte. ${ }^{29}$ Am 5. Juli 1203 machten sich die Kreuzfahrer daran diesen strategisch immens wichtigen Punkt zu erobern. Robert de Clari berichtet, wie sich das Heer am Morgen in Bewegung setzte: „Die Ritter gingen mit ihren Pferden an Bord der Lastschiffe und

\footnotetext{
${ }^{28}$ Niketas Choniates: Kreuzfahrer, S. 116.

29 Turnbull: Walls, S. $15 f$.
} 
fuhren los. Man ließ die Trompeten aus Silber und Erz, wohl mindestens hundert Paar, und auch die Trommeln und Pauken laut und stark erschallen." ${ }^{30}$ Die Venezianer griffen zur See an und schickten kleinere Barken mit Armbrust- und Bogenschützen voraus, um den Strand von den Griechen zu räumen und Platz für die Landung der Truppen zu machen. Als das geschafft war, gingen die restlichen Truppen an Land. „So landete die Flotte. Und als sie gelandet war, kamen die Ritter alle auf ihren Pferden heraus: Die Lastschiffe waren so gebaut, dass sie große Klappen hatten, die sich leicht öffnen ließen und aus denen man eine Landungsbrücke herauswarf, über welche die Ritter alle auf ihren Pferden sitzend an Land kommen konnten." 31

Um eine solche, bei Robert de Clari recht einfach anmutende, Landung durchzuführen, war in Wahrheit ein komplexes Wendemanöver zu meistern. Die Buganker der huissiers wurden noch vor Erreichen der Küste herabgelassen und das Schiff über sie hinweg gefahren. Dann musste das Heck zum Strand hin ausgerichtet werden, indem die eine Hälfte der Besatzung vorwärts, die andere rückwärts ruderte. Im richtigen Moment wurden dann Haltetaue ausgeworfen und die Landungsbrücken herabgelassen. Eine solches Manöver erforderte viel Erfahrung und unterstreicht die Bedeutung, die die gut eingeübten venezianischen Seeleute für den Erfolg der Landeoperation hatten. ${ }^{32}$ Nach kurzem, aber heftigen Kampf wurde Galata eingenommen und die Byzantiner flohen über einen Landungssteg nach Konstantinopel. Die Sperrkette wurde zerstört. Ihre Wirkung als Hafensperre war stets nur so effektiv gewesen, wie die, zu ihrer Verteidigung postierten Schiffe, ${ }^{33}$ deren Zustand bereits dargestellt wurde. Die lateinische Flotte konnte in das Goldene Horn fahren und dort bereiteten sich Kreuzfahrer und Venezianer auf den Sturm auf die Stadt vor.

Der Angriff auf Konstantinopel begann am 11. Juli. Da die Kreuzfahrer ihre Stärken eher in der Landkriegsführung sahen, wurde beschlossen, dass die Venezianer zur See, das restliche Heer aber die landeinwärts gerichtete, westlichen Stadtseite angreifen sollte. ${ }^{34}$ Die jeweiligen Fähigkeiten der verschiedenen Kontingente wurden hier für die Aufgabenverteilung berücksichtigt und klug verteilt. Die Kreuzfahrer brachten ihre Belagerungsmaschinen vor dem Blachernenpalast, am Nordzipfel der

\footnotetext{
30 Robert de Clari: Eroberung, S. 98.

31 Robert de Clari: Eroberung, S. 98.

32 Pryor; Wilson: Chain, S. 377.

33 Pryor/Wilson: Chain, S. 383.

${ }^{34}$ Robert de Clari: Eroberung, S. 100.
} 
Stadt, in Stellung und errichteten ein Lager, welches durch eine Holzpalisade vor Angriffen geschützt sein sollte.

Die häufigsten und effektivsten Belagerungsmaschinen waren die Blide und die Mangonelle, welche eine kleinere Version der erstgenannten Waffe war. Beides waren Wurfgeschütze, die Steine und andere Geschosse durch die Spannkraft einer Torsionswicklung am unteren Ende eines Wurfarms schleuderten. Sie bestanden aus einem langen Balken, der an einer Achse befestigt war. An einem Ende befand sich ein Gegengewicht, am anderen eine Schlinge, in der das Geschoss lag und dann in hohem Bogen geschleudert werden konnte. Die größten Bliden konnten Geschosse mit einem Gewicht von mehr als einer Tonne verschießen. ${ }^{35}$ Die hier eingesetzten Maschinen und vor allem die Mangonellen dürften allerdings deutlich kleiner gewesen sein. Robert de Clari zeigt sich besonders beeindruckt von der technischen Improvisationskunst der Venezianer, die sich hier als wahre Spezialisten bewiesen, wenn er berichtet: „Er [der Doge] ließ nämlich die Rahen, [...] mit starken Seilen an die Masten binden und machte so darüber feste Brücken und Geländer, ebenfalls mit Hilfe von Seilen. Sie waren so gemacht, daß drei gewappnete Ritter nebeneinander vorgehen konnten. Der Herzog ließ sie so gut schützen und an den Seiten mit dickem Segeltuch bedecken, daß diejenigen, die darüber gingen, um anzugreifen, sich nicht vor den Geschossen der Armbrustschützen und den Pfeilen fürchten mussten. [...] Auf jedem der Lastschiffe hatten sie eine Mangonelle, die ständig Steine gegen die Mauern und in die Stadt warf.“36

Dem gemeinsamen Angriff gingen einige Tage gegenseitiger Beschuss und kleinere Scharmützel voraus, bis am 17. Juli der Sturm auf die Stadt begann. Während die Venezianer an ihrer Stelle durchaus erfolgreich waren und mehrere Türme der Stadtverteidigung einnehmen konnten, wurden die Kreuzfahrer von dem aus der Stadt strömenden byzantinischen Heer unter der Führung Alexios III. angegriffen. Doch obwohl Villehardouin berichtet, dass die Feinde mit einer Überzahl von 60 Schlachthaufen gegenüber den nur 6 Schlachthaufen der Kreuzfahrer überlegen waren, kam es zu keinen ernsten Kampfhandlungen und der Kaiser zog sich, ohne ein Gefecht zu liefern, schnell wieder in die Stadt zurück. ${ }^{37}$ Zwar dürften Villehardouins Zahlenangaben wohl stark übertrieben sein, dennoch scheinen sich die Kreuzfahrer

\footnotetext{
35 Sean McGlynn: Art. Siege Warfare, in: The Oxford Encyclopedia of Medieval Warfare and Military Technology 3, Oxford 2010, S. 264-270, hier S. 266f., im Folgenden zitiert als McGlynn: Art. Siege Warfare.

36 Robert de Clari: Eroberung, S. 100.

37 Geoffroy de Villehardouin: Eroberung, S. 58.
} 
tatsächlich in zahlenmäßiger Unterlegenheit befunden zu haben. Um nicht von der Schlachtlinie der Byzantiner überflügelt zu werden, waren sie dazu gezwungen, ihr auch die Angehörigen des Versorgungstross' entgegenzustellen, welche teilweise nur mit Küchengeräten bewaffnet in die eigene Schlachtreihe rücken mussten. ${ }^{38}$ Die Venezianer wiederum konnten sich in der Stadt nicht halten und legten Feuer, bevor sie sich zurückzogen, um dem restlichen Heer gegen die Gefahr an Land zu helfen. Zwar war es nicht gelungen die Stadt zu erobern, jedoch verlor Alexios III. durch seinen kampflosen Rückzug den Rückhalt der Byzantiner, sodass er in der Nacht fliehen musste. Am 18. Juli öffneten sich die Tore Konstantinopels für die Kreuzfahrer. Die Stadt war erobert. ${ }^{39}$ Über die Gründe des kampflosen Rückzugs der Byzantiner kann nur gemutmaßt werden. Möglicherweise handelte es sich bei dem Ausfall um ein reines Ablenkungsmanöver, das die bereits in die Stadt eindringenden Venezianer dazu zwingen sollte, eigene Truppen vom Angriff abzuziehen und sie zur Verstärkung der Kreuzfahrer in den Norden zu schicken. Wahrscheinlicher ist jedoch, dass die Nachricht vom Einbruch der Venezianer Alexios III. dazu veranlasste, seine Soldaten wieder zurück in die Stadt zu führen. ${ }^{40}$ Vielleicht hatte den jungen und unerfahrenen Heerführer aber auch nur der Mut verlassen.

Obwohl Alexios IV. anfänglich durchaus bemüht war, die versprochenen Leistungen an die Kreuzfahrer zu zahlen und dafür sogar Kirchengüter einschmelzen ließ, sah er sich doch bald außer Stande den Zahlungen weiter nachzukommen. Bald stoppten die Zahlungen gänzlich. Dies führte zu erneuter Feindschaft zwischen den Kreuzfahrern und Byzanz. ${ }^{41}$ Eines Nachts schickten die Byzantiner plötzlich Brander, kleine mit trockenem Holz und Speck beladene Schiffe, die in Brand gesetzt und in Richtung einer feindlichen Flotte fahren gelassen wurden, gegen die venezianischen Schiffe. Es wurde kaum Schaden angerichtet, aber nun wurde endgültig beschlossen die Stadt erneut zu erobern. ${ }^{42}$ Den Byzantinern wurde immer wieder der Besitz einer Waffe namens ,Griechisches Feuer' nachgesagt, obwohl es fraglich scheint, ob sie 1204 darüber verfügten. Dabei handelte es sich um eine Vorrichtung, die ein hochentzündliches Ölgemisch verschießen konnte, ähnlich einem modernen Flammenwerfer. Mit Wasser ließ sich ein so entfachtes Feuer nicht löschen, was die Waffe besonders gefürchtet machte. Die Westeuropäer verwendeten den Namen

\footnotetext{
38 Kolias: Aspects, S.129.

${ }^{39}$ Nicolle: Crusade, S. 63.

40 Kolias: Aspects, S. 134.

41 Geoffroy de Villehardouin: Eroberung, S. 66-68.

42 Robert de Clari: Eroberung, S. 110f.
} 
jedoch oft auch für andere Brandwaffen. ${ }^{43}$ Der von Robert de Clari überlieferte Einsatz von einfachen Brandern anstelle einer so hochentwickelten Waffe legt zudem die Vermutung nahe, dass entweder die Kenntnisse oder die Rohstoffe zur Anwendung des ,Griechischen Feuers' im 13. Jahrhundert den Byzantinern nicht mehr zur Verfügung standen.

Am 9. April erfolgte der zweite Angriff auf die Stadt. Mittlerweile war Alexios IV. bei einem Putsch ermordet worden und der neue Kaiser Alexios V. befehligte die Verteidigung. Beide Seiten hatten sich wochenlang vorbereitet. Während die Byzantiner ihre Mauern beträchtlich erhöht hatten, ${ }^{44}$ berichtet Robert de Clari von den Vorkehrungen der Kreuzfahrer: „Und die Venezianer ließen die Enterbrücken auf ihren Schiffen wieder herrichten, und die Franzosen ließen andere Kriegsmaschinen bauen, die man „Katzen“ nennt, und „Gerippe“ und „Säue“, um die Mauern zu unterminieren.“45 ,Katzen' und ,Säue' waren fahrbare Schutzhütten, die als Deckung für Rammböcke und Minierarbeiten an der Mauer dienten. Bei der Unterminierung wurde ein Tunnel unter die Mauer gegraben. Im richtigen Moment wurden dann die Stützen des Tunnels verbrannt und die Mauer sollte durch die entstandene Unterhöhlung in sich zusammenbrechen. ${ }^{46}$ Auch Choniates beschreibt den Anblick der feindlichen Flotte: „Die Fahrzeuge hatten sie mit Rindshäuten umgeben, damit sie nicht durch Feuer zerstört werden könnten. Und an Kranarmen hatten sie Strickleitern befestigt [...]. " ${ }^{47}$ Die von Choniates erwähnten Kuhhäute waren ein bewährtes Mittel, um die eigene Kriegsmaschinerie vor Brandwaffen zu schützen. Sie wurden in Essig oder andere nicht entflammbare Flüssigkeiten getränkt ${ }^{48}$ und dann um die hölzernen Teile der Schiffe und Belagerungsmaschinen gelegt. ${ }^{49}$ Trotz der sorgfältigen Vorbereitungen schlug der Angriff fehl und wurde deshalb am 12. April an selber Stelle wiederholt. Der Vorschlag der Franzosen, an anderer Stelle anzugreifen, wurde von den Venezianern abgelehnt, da nur an der bereits attackierten Stelle die Strömung des Meeres einen Angriff zuließ. ${ }^{50}$ Ohne die nautischen Kenntnisse der Venezianer wäre wohl also auch der nächste Angriff gescheitert.

\footnotetext{
${ }^{43}$ Alexandru Madgearu: Art. Greek Fire, in: The Oxford Encyclopedia of Medieval Warfare and Military Technology 2, Oxford 2010, S. 221-222.

44 Kolias: Aspects, S. 133.

45 Robert de Clari: Eroberung, S. 117.

46 McGlynn: Art. Siege Warfare, S. 268f.

${ }^{47}$ Niketas Choniates: Kreuzfahrer, S. 119.

48 McGlynn: Art. Siege Warfare, S. 267.

49 Kolias: Aspects, S. 132.

50 Geoffroy de Villehardouin: Eroberung, S. 75.
} 
Beim zweiten Angriff gelang es den Kreuzfahrern schließlich von den Schiffen aus, die Mauer zu erobern. Der erste, der einen Turm von einem venezianischen Schiff aus erobern konnte, war ein französischer Ritter. Ein nur leicht gerüsteter Venezianer, der vor ihm den Turm bestieg, wurde bei dem Versuch getötet. ${ }^{51}$ Hier zeigte sich die schwere Rüstung der Ritter als entscheidender Vorteil im Nahkampf. Auch die untere Mauer wurde von einem französischen Ritter namens Pierre D'Amiens und dem Bruder von Robert de Clari durchbrochen. Zumindest Pierre D'Amiens Rolle dabei wird auch von Choniates bestätigt: „Es war ein Mann, den man für fähig gehalten hätte, ganze Heere vor sich herzutreiben. An Größe glich er beinahe jenen neun Klafter langen Giganten und sein Helm war gebildet wie eine turmbewehrte Stadt."52

Nachdem die Verteidigungsanlagen gefallen waren, verfielen die Griechen in eine ungeordnete Flucht und selbst Alexios V. persönlich konnte sie nicht mehr dazu bewegen weiterzukämpfen. Was nun folgte, war die vollkommene Plünderung der Stadt durch die Kreuzfahrer. Choniates beschreibt die Situation folgendermaßen: „Die Feinde stürmten, da niemand ihnen entgegentrat, hierhin und dorthin und zogen das Schwert gegen jegliches Alter und Geschlecht [...]." ${ }^{\text {"53 }}$ Ein weiteres fatales Feuer brach in der Nacht aus und zerstörte große Teile der Stadt. Konstantinopel war nun endgültig gefallen und Villehardouin vermittelt den letzten Eindruck dieses Kampfes: „Es gab da so viele Tote und Verwundete, daß derer kein Ende und kein Maß war." 54

Der Vierte Kreuzzug verfehlte sein eigentliches Ziel, die Rückeroberung Jerusalems von den Muslimen, vollkommen. Die Heilige Stadt war zu keinem Zeitpunkt durch das Kreuzfahrerheer gefährdet. Militärisch und finanziell endete er für die Kreuzfahrer jedoch als Erfolg, auch wenn die neuen Besitzungen zum Teil in nur wenigen Jahren wieder verlorengingen. Das Kreuzfahrerheer profitierte erheblich von seiner ,multinationalen' Zusammensetzung, die dazu führte, dass jedes Kontingent seine persönlichen Stärken in die Unternehmung miteinfließen lassen konnte. Besonders die Rolle der Venezianer kann wohl nicht überschätzt werden. Die venezianische Flotte machte die gesamte Reise und schließlich auch die Eroberung Konstantinopels erst möglich und ohne ihre seefahrerischen Kenntnisse wäre wohl auch der zweite Versuch die Stadt am 12. April 1204 zu erobern fehlgeschlagen. Ohne das

\footnotetext{
${ }^{51}$ Robert de Clari: Eroberung, S. 120.

52 Niketas Choniates: Kreuzfahrer, S. 145.

53 Niketas Choniates: Kreuzfahrer, S. 146.

${ }^{54}$ Geoffroy de Villehardouin: Eroberung, S. 76.
} 
Wissen um die örtlichen Strömungen hätten die Kreuzfahrer wohl an anderer Stelle angegriffen und wären aller Wahrscheinlichkeit nach abgetrieben und abermals besiegt worden. Ob dem Heer die Kräfte zu einem dritten Angriff dann noch zur Verfügung gestanden hätten, kann durchaus bezweifelt werden. Interessanter Weise waren es allerdings die französischen Kreuzfahrer, die den Durchbruch nach Konstantinopel schafften. Die Technologie Venedigs hatte sie an bzw. auf die Mauern der Stadt gebracht. Doch diese zu erobern und zu halten gelang den nur leicht gerüsteten Venezianern im Kampf mit der byzantinischen Elitegarde nicht. Erst die schwer gerüsteten französischen Ritter schafften es, die Verteidigungsanlagen zu überwinden und zu behaupten. Der Erfolg des Kreuzzuges hing also im erheblichen Maße von dem Zusammenspiel der Kompetenzen der einzelnen Teilnehmer ab. Jeder für sich hätte entweder nicht die Stärke oder die technischen und logistischen Fähigkeiten gehabt, die Angriffe auf Konstantinopel zu einem erfolgreichen Ende zu führen.

Für die Byzantiner endete der Kreuzzug in einer Katastrophe. Zwar standen ihnen während der Kämpfe auch technologisch fortschrittliche Waffen, in der Belagerungskriegsführung erfahrene Soldaten sowie Elitetruppen wie die Warägergarde zur Verfügung. Jedoch mangelte es entweder an numerischer oder moralischer Stärke, um die Kreuzfahrer abzuwehren, möglicherweise traf beides zu. Gepaart mit dem schwächlichen Führungsstil des Kaisers Alexios III. und vielleicht auch der Inkompetenz anderer militärischen Führer und Berater bedeutete dies von Beginn an schlechte Chancen, die auch der energischere Alexios V. nicht ausreichend wettmachen konnte. 